\title{
Time- and space-resolved dynamics of melting, ablation, and solidification phenomena induced by femtosecond laser pulses in germanium
}

\author{
Jörn Bonse, ${ }^{*}$ Guillaume Bachelier, ${ }^{\dagger}$ Jan Siegel, and Javier Solis ${ }^{\ddagger}$ \\ Laser Processing Group, Instituto de Optica, C.S.I.C., Serrano 121, E-28006 Madrid, Spain \\ (Received 17 February 2006; revised manuscript received 30 May 2006; published 6 October 2006)
}

\begin{abstract}
Femtosecond time-resolved microscopy has been used to analyze the structural transformation dynamics (melting, ablation, and solidification phenomena) induced by intense $130 \mathrm{fs}$ laser pulses in single-crystalline (100)-germanium wafers on a time scale from $\sim 100$ fs up to $10 \mathrm{~ns}$. Complementary information on longer time scales $(350 \mathrm{ps}-1.4 \mu \mathrm{s})$ has been obtained by means of simultaneous streak camera and photodiode measurements of the sample surface reflectivity. In the ablative regime, transient surface reflectivity patterns are observed by fs microscopy on a ps to ns time scale as a consequence of the complex spatial density structure of the ablating material. Complementing point-probing streak camera measurements allow one to characterize the temporal evolution in real time up to $40 \mathrm{~ns}$ after the fs-laser pulse excitation. Fs microscopy reveals additional reflectivity patterns for fluences below the ablation threshold of the germanium. It is shown that these patterns are originating from the selective removal of the native oxide layer at the wafer surface within a certain fluence range. After solidification, and in contrast to other semiconductors, surface amorphization has not been observed in (100)-germanium upon femtosecond laser pulse irradiation in the studied fluence range.
\end{abstract}

DOI: 10.1103/PhysRevB.74.134106

PACS number(s): 79.20.Ds, 78.47.+p, 64.70.-p

\section{INTRODUCTION}

Germanium $(\mathrm{Ge})$ is a group IV semiconductor with a high carrier mobility that has been widely used in solid-state electronics. Due to its small bandgap energy, Ge can be optically excited with infrared and near infrared radiation which makes it useful for detection and modulation applications. ${ }^{1}$ Indirect interband transitions between the valence and the conduction band require a minimum photon energy of $0.66 \mathrm{eV}$, whereas at least $0.80 \mathrm{eV}$ are needed for a direct transition. $^{2}$

Several studies have been devoted to the interaction of laser radiation with this semiconductor for laser energy densities (fluences) below the damage threshold. ${ }^{3-5}$ Close to and above this threshold there have been a number of studies investigating the dynamics of phase transitions such as melting, ablation, or rapid resolidification phenomena in the picosecond to microsecond temporal scale. ${ }^{4,6-10} \mathrm{Up}$ to now, very few studies are available regarding the dynamics of ultrafast phase transitions in germanium induced by femtosecond laser pulses. ${ }^{11-13}$

In this paper we report a detailed study of the melting, ablation, and resolidification dynamics of single-crystalline germanium $(c-\mathrm{Ge})$ under femtosecond laser pulse irradiation using time- and space-resolved reflectivity measurements. Femtosecond time-resolved microscopy has already been used in investigations of laser-induced phase transitions in other semiconductors such as carbon (C), ${ }^{14}$ silicon $(\mathrm{Si}),{ }^{15,16}$ or the III-V compound material gallium arsenide (GaAs). ${ }^{13,17,18}$ In this pump and probe technique each laser exposure provides a single data point on a time axis. By means of performing multiple measurements the time evolution of the signal can be reconstructed. Due to practical limitations in the use of optical delay lines, this technique has been typically used for delays up to a few nanoseconds, whereas typical solidification processes (crystallization/ amorphization) can occur on much longer time scales. In contrast to the pump and probe approach which is usually referred to as a time-resolved technique, so-called real-time measurements can provide the complete time evolution of the signal at a single sample location using a single laser pulse exposure. ${ }^{19}$ As a consequence, this approach is very robust to sample inhomogeneities and laser pulse fluctuations. The detectors used are either fast photodiodes or streak cameras, depending on the desired time window and temporal resolution.

Our study, combining both approaches and covering a time span from $\sim 100$ fs up to a few $\mu$ s, provides detailed insights into the dynamics of melting and ablation as well as the subsequent resolidification phenomena, using both a fstime-resolved microscopy and complementary real-time streak camera and photodiode measurements. Taking benefit from the imaging capabilities of our setup, a phenomenon of transient surface wave patterns due to the selective removal of the native oxide layer is reported for the melting regime of femtosecond laser pulse irradiated germanium when a certain fluence threshold is exceeded.

\section{EXPERIMENTAL METHODS}

The data reported here were obtained on polished (100) crystalline Ge wafers ( $p$-doped, $0.02 \Omega \mathrm{cm}$ ). Before irradiation, successive ultrasonic bath cleaning in different organic solvents (trichloroethylene, acetone, and ethanol) was performed. No attempt was made to remove the native oxide layer of $3 \pm 0.3 \mathrm{~nm}$ thickness (determined by variable angle spectral ellipsometry).

The laser used for irradiation was a chirped pulse regenerative amplification Ti:sapphire laser system (Spectra Physics, Spitfire) providing linearly polarized pulses of $\sim 130 \mathrm{fs}$ duration at a center wavelength of $800 \mathrm{~nm}$. Since at this wavelength the photon energy of $1.55 \mathrm{eV}$ is sufficient to in- 


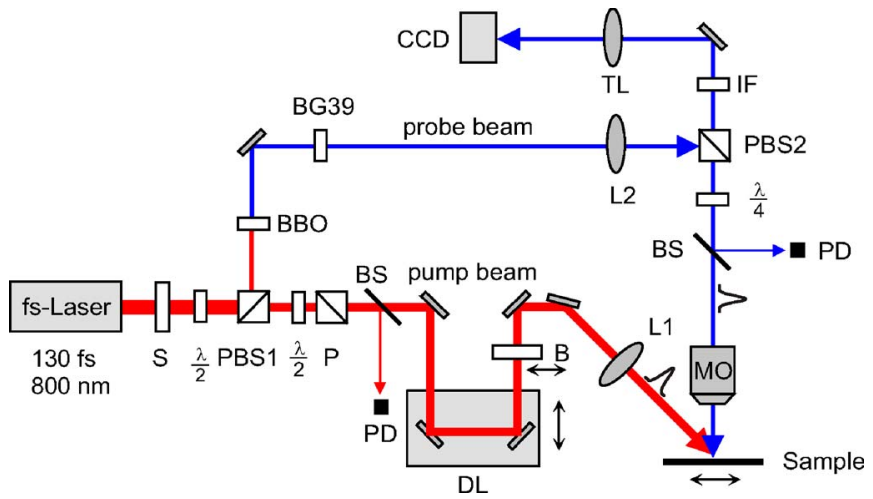

FIG. 1. (Color online) Scheme of the experimental fs-TRM setup. Abbreviations: B-beam stop; BBO-nonlinear crystal; BG39-bandpass filter; BS — beam splitter; CCD — camera; DLdelay line; IF-interference filter; $\frac{\lambda}{2}$-halfwave-plate; $\frac{\lambda}{4}$-quarterwave-plate; L-lens; $\mathrm{MO}$-microscope objective; $\mathrm{P}$-Glan laser polarizer; PBS—polarizing beam splitter; PD— photodiode; $\mathrm{S}$-electromechanical shutter; TL—tube lens.

duce a direct band transition, this mechanism is expected to be dominant in the vicinity of the $\Gamma$ point of the band structure. The resulting optical penetration depth $1 / \alpha$ at low intensities is approximately $200 \mathrm{~nm}$ (Ref. 20). The irradiation of the sample was performed in air at atmospheric pressure. Each surface region was irradiated only once in order to induce structural modifications.

\section{A. Fs-time-resolved microscopy (fs-TRM)}

The temporal evolution of the surface reflectivity upon irradiation of the sample was measured using a $f$ s-timeresolved microscopy (fs-TRM) setup that provides the capability of imaging the laser-excited surface for variable time delays (up to $10 \mathrm{~ns}$ ) between the surface exciting pump and the illuminating probe pulses.

The fs-TRM experiment was based on a noncollinear geometry $^{16}$ with different wavelengths for the pump $(800 \mathrm{~nm})$ and probe $(400 \mathrm{~nm})$ pulses (see Fig. 1). An electromechanical shutter $(\mathrm{S})$ allowed a single laser pulse to be selected from a continuous pulse-train $(100 \mathrm{~Hz}$ pulse repetition rate) provided by the regenerative amplifier system. The selected laser pulse was then divided into pump and probe pulses using a combination of a halfwave-plate $\left(\frac{\lambda}{2}\right)$ and a polarizing beam splitter cube (PBS1) for adjusting the energy ratio between them.

Another combination of a halfwave-plate $\left(\frac{\lambda}{2}\right)$ and a Glan laser polarizer $(\mathrm{P})$ was used in the pump beam for variable energy attenuation. After passing through an optical delay line (DL), the pump pulse was focused by a lens (L1) at an angle of incidence of $54^{\circ}$ onto the sample surface, being $s$ polarized with respect to the plane of incidence. At the surface, the elliptical Gaussian spot showed a diameter $\left(1 / e^{2}\right.$ decrease) of $2 w_{0, x} \sim 100 \mu$ and $2 w_{0, y} \sim 60 \mu \mathrm{m}$ along its long and short axis, respectively.

The low intensity probe beam (previously split off from the PBS1), was frequency doubled by means of a nonlinear crystal (BBO). The residual $800 \mathrm{~nm}$ radiation was eliminated by an IR-cutting bandpass filter (Schott, BG39). The probe beam was then focused by a lens (L2) to the back-focal plane of a long-working distance microscope objective (MO: Mitutoyo, M-Plan-NIR, $20 \times, \mathrm{NA}=0.4)$ after reflection in a polarizing beam splitter cube (PBS2). A quarterwave-plate $\left(\frac{\lambda}{4}\right)$ was used to convert the linear polarization state into a circular one. The MO finally recollimates this beam such that it can be used for normal incidence illumination of the sample area which is excited by the $800 \mathrm{~nm}$ pump beam.

The probing $400 \mathrm{~nm}$ radiation reflected at the sample surface was then reconverted to a linear polarization state of orthogonal orientation by passing a second time through the same $\frac{\lambda}{4}$ plate. The probe beam, capable thus to pass through PBS2, was used directly to image the laser-excited surface region by means of the $\mathrm{MO}$ and an additional tube lens (TL, $f=200 \mathrm{~mm}$ ) onto a CCD-camera (PCO, Pixelfly HiRes, image acquisition time $100 \mu \mathrm{s}$ ), which was computer controlled and synchronized with the laser system. In order to separate the imaging radiation from scattered light of the pump beam or light originating from optical plasma emissions during the ablation process, a narrow band interference filter (IF) with a center wavelength of $400 \mathrm{~nm}$ and a bandwidth of $10 \mathrm{~nm}$ was additionally placed in the imaging path.

The reflectivity measurements were performed with a probe beam fluence well below the melting threshold, whereas the fluence in the pump beam was sufficient to induce phase transitions such as melting or ablation. The pump pulse energies were measured by means of a photodiode (PD) calibrated against a pyroelectric detector at the sample site.

At each sample position, two measurements had been used to determine the laser-induced reflectivity change at the chosen delay time $\tau$. The first measurement [with the pump pulse blocked by the beam stop (B)] was used to obtain a reflectivity image $R_{0}(x, y)$ of the sample surface at the measurement position before exposure of the pump pulse, whereas the second allowed the recording of the surface reflectivity image $R(\tau, x, y)$ upon pump pulse excitation. The normalized reflectivity change $\frac{\Delta R}{R}(\tau, x, y)=\frac{R(\tau, x, y)-R_{0}(x, y)}{R_{0}(x, y)}$ has then been calculated by subtracting both images and by normalizing by the reflectivity in the undisturbed surface. In this way, the image normalization procedure allows one to take into consideration the spatial profile of the illumination probe beam as well as variations in the surface reflectivity due to contaminants, small surface imperfections, etc.

Apart from the two images of the nonirradiated surface and the transient image at the chosen delay time, an additional image has been acquired at the same sample site several seconds after the irradiation with the pump pulse in order to reveal the permanent surface modifications. In the following, those final images are indicated by the symbol " $\infty$ ".

The estimated uncertainty in $\Delta R / R$ at this probing wavelength is $\sim 0.05$. The temporal resolution of the fs-TRM setup has been determined to be approximately $400 \mathrm{fs}$.

\section{B. Real-time reflectivity measurements (RTR)}

In order to analyze the surface reflectivity changes over longer time scales from the ps- to the $\mu$ s-range, a comple- 


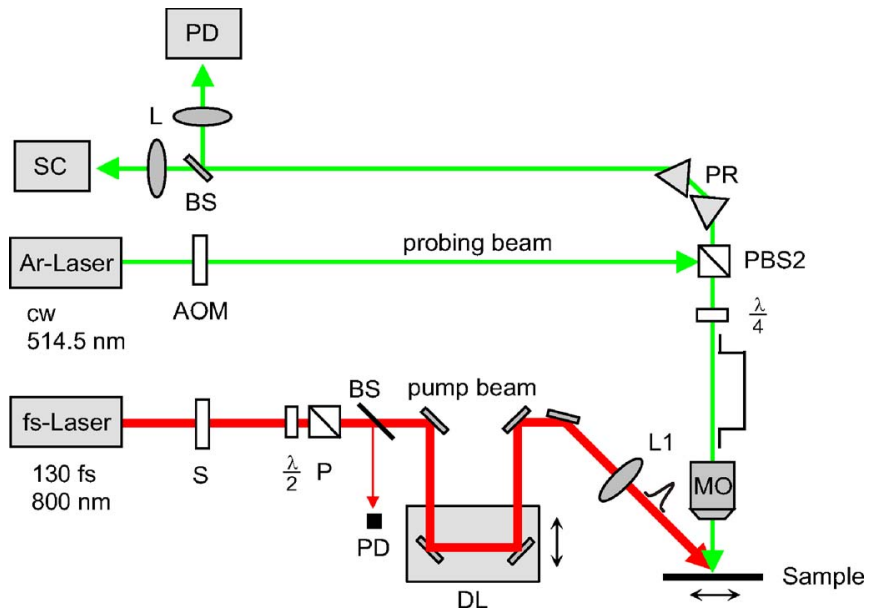

FIG. 2. (Color online) Scheme of the experimental RTR setup. Abbreviations: AOM - acousto-optical modulator; BS-beam splitter; DL-delay line; $\frac{\lambda}{2}$ —halfwave-plate; $\frac{\lambda}{4}$-quarterwave-plate; L-lens; $\mathrm{MO}$-microscope objective; $\mathrm{P}$-Glan laser polarizer; PBS—-polarizing beam splitter; $\mathrm{PD}$ - photodiode; $\mathrm{PR}$ - prism pair; $\mathrm{S}$-electromechanical shutter; SC-streak camera.

mentary approach has been used (Fig. 2). It is essentially based on the fs-TRM setup and can be used with small modifications to perform real-time reflectivity measurements (RTR) by means of a streak camera (Hamamatsu Model C5680, equipped with a single-sweep unit Model M5676; time resolution of $350 \mathrm{ps}$ in a time window $50 \mathrm{~ns}$ ) and a fast photodiode coupled to an oscilloscope (time resolution of a few ns).

In that approach, the $400 \mathrm{~nm}$ fs-probe beam was substituted by a continuous wave (cw), single mode and singlefrequency probing $\mathrm{Ar}^{+}$-laser beam $(514.5 \mathrm{~nm})$, modulated by means of an acousto-optical modulator (AOM) to produce a single rectangular pulse of $2 \mu$ s duration. This probe pulse was synchronized with the fs-laser pulse. In order to perform a point-probing measurement in the center of the fs-pumped area, the lens (L2) in the probe beam path, focusing to the back-focal plane of the MO, was removed generating a focused Gaussian beam with a diameter of $2 w_{0} \sim 0.9 \mu \mathrm{m}$ at the sample surface. Hence, a pump-to-probe spot size ratio of $\sim 110$ was used experimentally. The back-reflected probing signal was recollimated by the $\mathrm{MO}$ and analyzed simultaneously by means of the streak camera (SC) and the photodiode (PD). For that purpose, a spectral separation of the monochromatic probing $\mathrm{Ar}^{+}$laser beam and other radiation contributions, such as light of the fs-pump beam scattered from the surface upon irradiation or possible subsequent optical plasma emissions, was performed using a prism pair (PR) just after PBS2. As in the case of fs-TRM, the normalized surface reflectivity change $\Delta R / R$ has been calculated using the reflectivity value of the undisturbed surface. The uncertainty in $\Delta R / R$ are $\sim 0.04$ and $\sim 0.03$ for the streak camera and the photodiode measurements, respectively. Additional details regarding the temporal characteristics of the probing laser beam and the photodetection systems (PD, SC) can be found in Ref. 19.

\section{RESULTS AND DISCUSSION}

\section{A. Fs-laser-induced ablation}

\section{1. $f s-T R M$}

A series of fs-TRM images obtained on a germanium surface is shown in Fig. 3 for an excitation peak fluence in the ablative regime $\left(\Phi_{0}=3.10 \pm 0.08 \mathrm{~J} / \mathrm{cm}^{2}\right)$, well above the ab-
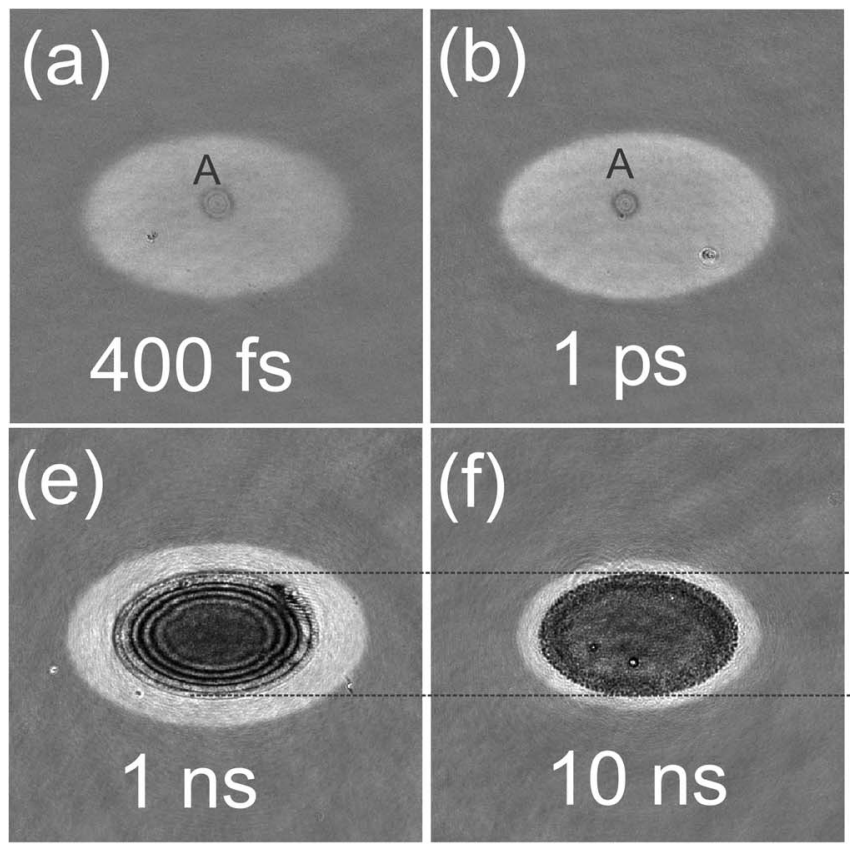
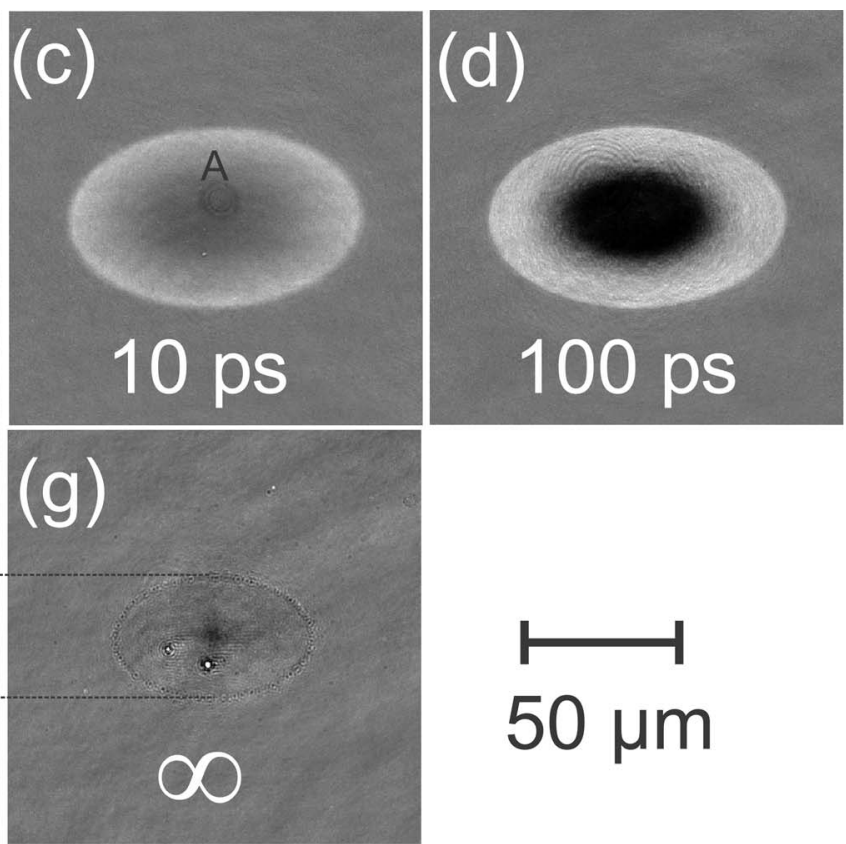

FIG. 3. Images of a germanium surface at different times after the exposure to the pump pulse (pump fluence $3.10 \pm 0.08 \mathrm{~J} / \mathrm{cm}^{2}$, ablative regime). A denotes an imaging artifact which is not related to a modification of the sample surface. The image sequence is encoded in a linear gray scale with an optimized contrast. 
lation threshold fluence $\left(\Phi_{a b l}=1.32 \mathrm{~J} / \mathrm{cm}^{2}\right)$. The series covers the entire period from shortly after the end of laser energy deposition up to the appearance of the final surface structure. The number in each frame indicates the delay time between the pump and the probe pulse. Because of the large angle of $54^{\circ}$ between the pump beam and the imaging probe beam, the laser excited area has an elliptical shape. Note that the small dark feature (indicated by " $A$ ") in Figs. 3(a)-3(c) is an imaging artifact, arising from an optical reflection in the setup in conjunction with the image normalization procedure. It is not related to any transient physical process at the sample surface.

At early times, immediately after the arrival of the pump pulse to the surface (400 fs delay), an increase of the surface reflectivity in the center of the irradiated spot can be seen [Fig. 3(a)]. This is due to the strong electronic excitation resulting in the formation of an electron-hole plasma, which can destabilize the lattice structure on a subpicosecond time scale when a critical electron density between $10^{21}$ and $10^{22} \mathrm{~cm}^{-3}$ in the conduction band is exceeded. ${ }^{21}$ This ultrafast phase transition is usually referred to as nonthermal melting and has already been reported in germanium ${ }^{11,12}$ and also in other semiconductors. ${ }^{17,22-24}$

Already after $1 \mathrm{ps}$, the reflectivity of the entire irradiated region has increased to almost the same reflectivity value as for the center of the image for 400 fs delay time, giving rise to the appearance of a bright elliptical area with a sharp defined edge [Fig. 3(b)]. It is associated with the formation of an optically thick layer of liquid metallic germanium $(\ell-\mathrm{Ge})$ on the surface. Note that the optical penetration depth of the $400 \mathrm{~nm}$ probe radiation in $\ell$-Ge is only $7-8 \mathrm{~nm}^{25}$

On a time scale of tens of picoseconds, the reflectivity of almost the entire spot is lowered [Fig. 3(c)], which might arise from the optical properties of a superheated liquid. ${ }^{17,26}$

After some tens to hundreds of picoseconds, an even darker appearing zone of strongly decreased reflectivity can be seen in the central region of the spot [Fig. 3(d), 100 ps delay]. It is indicative of the onset of ablation. At later times a characteristic pattern of rings develops from this dark central feature [Fig. 3(e), 1 ns delay]. Additional images taken for delay times between $100 \mathrm{ps}$ and $1 \mathrm{~ns}$ (not shown here) reveal that these fringes are moving from the center towards the outer regions of the spot for increasing delays while increasing their number and decreasing their radial spacings. The nature of these rings has been already associated with an optical interference effect (dynamically moving Newton fringes). ${ }^{16,27}$ The corresponding interference fringes are visible during the formation and spatial movement of a complex material density profile as a consequence of the formation of a so-called rarefaction wave (and its subsequent reflection, when material is passing through the liquid-gas coexistence regime $^{28}$ ) after fs irradiation of semiconductors and metals. The resulting density profile consists, at the air side of the expanding material, of a thin ablating layer with nearly solid state material density and a thickness smaller than the optical penetration depth in this optically excited ablating layer. ${ }^{28,29}$ The partial reflections at this dynamically moving layer and the reflection at the remaining surface underneath then allows interference effects to occur transiently.

After $10 \mathrm{~ns}$ delay, fringes cannot be observed anymore in the fs-TRM images [Fig. 3(f)]. Their disappearance can

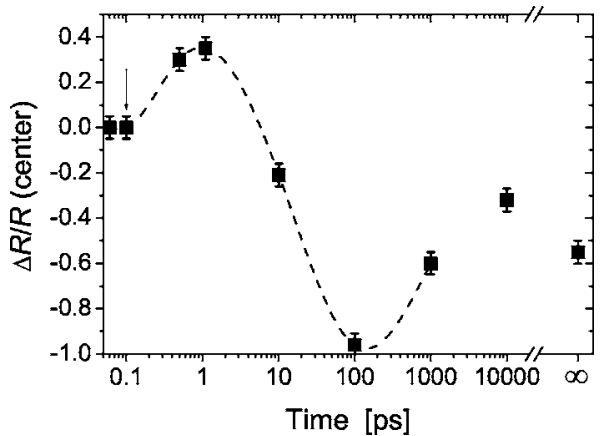

FIG. 4. Normalized surface reflectivity change $\Delta R / R$ as a function of time as measured in center of the irradiated regions shown in Fig. 3. Note the logarithmic time axis. The true zero delay is marked by an arrow. The final reflectivity change $(\Delta t=\infty)$ is marked on the right-hand horizontal axis. The line is to guide the eye.

arise, for instance, from the gradual flattening of the initially sharp density profile of the ablating layer. ${ }^{13}$ Another possible reason for this behavior is that the distance of the interface of the expanding ablated material to the surface has reached a value larger than half the coherence length of the fs-probe beam radiation $(\approx 15-20 \mu \mathrm{m})$, thus making interference impossible. The RTR streak camera measurements presented in Sec. III A 2 will provide more detailed insights into the ablation dynamics on the ns time scale and will give additional clues regarding the origin of the disappearance of the fringes.

Instead of the fringes, an irregularly shaded central region of reduced reflectivity can be seen then which is surrounded by an annulus of high reflectivity [Fig. 3(f)]. The latter indicates that the material is still molten in that area. Note that the outer diameter of the molten ring has already reduced its size when compared to earlier times [Figs. 3(b)-3(e)]. This indicates that the resolidification, which has started from the outer and deeper lying regions of the melt pool, becomes visible at the surface already after some nanoseconds. The outer edge of the dark central region coincides with the borders of a crater observable in the final surface morphology, as indicated by two horizontal lines [Fig. 3(g)]. However, the reduced reflectivity in the center of Fig. 3(f) when compared to the final image $(\mathrm{g})$ clearly evidences that after $10 \mathrm{~ns}$ the probe beam radiation is still interacting with ablating material.

To quantitatively follow the time evolution of the reflectivity, we have plotted in Fig. 4 the normalized surface reflectivity change $\Delta R / R$ as a function of the delay time, as evaluated in the center of the spots shown in Fig. 3. This representation is the equivalent to a point-probing conventional fs-pump-probe experiment and provides complementary information to RTR measurements but on much shorter time scales.

All discussed stages of nonthermal melting, ablation, and a partial recovery of the reflectivity can be seen in Fig. 4. The initial reflectivity increase on the subpicosecond to picosecond scale reaches values of $\Delta R / R \sim 0.35$. The onset of ablation after tens of ps then reduces the normalized reflectivity change to values approaching -1.0 after $100 \mathrm{ps,} \mathrm{which}$ means that the probe beam radiation is completely absorbed in the excited surface region. After 1 nanosecond, a partial 


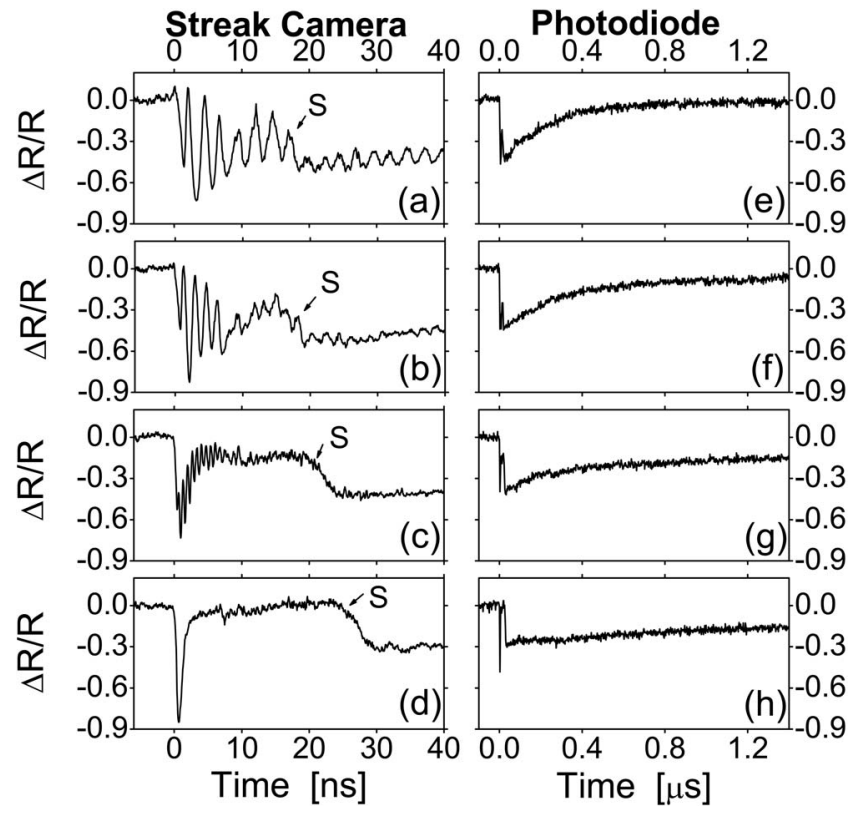

FIG. 5. Normalized surface reflectivity change as a function of time as measured with streak camera (a)-(d) and with a photodiode (e)-(h) at four different fluence levels $\left[(\mathrm{a})+(\mathrm{e}): 1.35 \mathrm{~J} / \mathrm{cm}^{2}\right.$, (b) $\left.+(\mathrm{f}): 1.53 \mathrm{~J} / \mathrm{cm}^{2},(\mathrm{c})+(\mathrm{g}): 1.68 \mathrm{~J} / \mathrm{cm}^{2},(\mathrm{~d})+(\mathrm{h}): 2.67 \mathrm{~J} / \mathrm{cm}^{2}\right]$.

reflectivity recovery is seen, finally leading to a permanent normalized reflectivity change of -0.55 . Note that the complete reflectivity dynamics especially on time scales longer than $1 \mathrm{~ns}$ is not resolved in our fs-TRM experiments here, which underlines the benefit of complementary pointprobing RTR measurements covering those longer time scales.

\section{Ps- and ns-RTR}

The ablation process has been studied on longer time scales (ns- $\mu$ s) using the RTR setup. Figure 5 shows the temporal evolution of the normalized surface reflectivity change as probed simultaneously by the streak camera $[(\mathrm{a})-(\mathrm{d})]$ and the photodiode $[(\mathrm{e})-(\mathrm{h})]$ in the center of the irradiated region upon irradiation at four different pump peak fluence levels up to two times the ablation threshold fluence. At a fluence $\Phi_{0}=1.35 \mathrm{~J} / \mathrm{cm}^{2}$, slightly above the ablation threshold [Fig. $5(\mathrm{a})$ ], the normalized reflectivity change shows rapid oscillations that last for more than 40 nanoseconds. They are associated with the outward moving dense shell of ablated material, which has already been observed in the fs-TRM (Fig. 3). This is a direct observation of the outward moving dense shell of ablating material for time scales up to 40 nanoseconds as well as in real time for a single laser pulse.

As seen in Figs. 5(a)-5(c), the period of the reflectivity oscillation $\Delta t$ strongly depends on the laser fluence. For a fluence level of $1.35 \mathrm{~J} / \mathrm{cm}^{2}$ [Fig. 5(a)] an average oscillation period of $2.6 \mathrm{~ns}$ is found, whereas at a higher level of $1.68 \mathrm{~J} / \mathrm{cm}^{2}$ it lasts only $0.7 \mathrm{~ns}$ [Fig. 5(c)]. The shortest oscillation period of $0.5 \mathrm{~ns}$ was observed at $2.08 \mathrm{~J} / \mathrm{cm}^{2}$ (data not shown here). These quantitatively measured values for $\Delta t$ provide a way for the estimation of the speed of the outward moving dense shell of material. ${ }^{49}$ For that we assume that it consists of a layer with constant density and thickness which initially propagates away from the remaining surface at constant velocity $v=\Delta x / \Delta t,{ }^{30}$ thus leaving behind a gas-filled or bubblelike space with a real part of the refractive index close to 1 (conditions already used and discussed in more detail in Refs. 18 and 28). The assumption of a constant velocity is justified by the experimental results shown in Fig. 5: The period of the reflectivity oscillations is constant [e.g., in Fig. $5(\mathrm{a})$ ], indicating that the speed of the shell of ablating material is constant at least during the first tens of nanoseconds. Then, for adjacent temporal reflectivity maxima or minima, an interference condition $2 \times \operatorname{Re}(n) \times \Delta x=1 \times \lambda$ should hold for the probing wavelength $\lambda=514.5 \mathrm{~nm}$. Hence, the average velocity can be calculated via $v=\lambda /[2 \operatorname{Re}(n) \times \Delta t]$ $\approx \lambda /(2 \Delta t)$. This simple relation provides values between $100 \mathrm{~m} / \mathrm{s}\left(1.35 \mathrm{~J} / \mathrm{cm}^{2}\right)$ and $515 \mathrm{~m} / \mathrm{s}\left(2.08 \mathrm{~J} / \mathrm{cm}^{2}\right)$.

In contrast to the fs-TRM measurements, a continuous wave argon laser is used here to probe the reflectivity dynamics of the surface. Hence, the coherence length of the laser beam plays no role in the disappearance of the fringes, as it may in the case of fs microscopy. The RTR technique allows one then to evaluate the "lifetime" of the ablating layer, i.e., the time during which the density profile is sufficiently sharp in the propagation direction and homogeneous in the perpendicular direction to allow interference effects to be observed. As shown in Figs. 5(a)-5(d), this lifetime strongly decreases with increasing fluences from more than $40 \mathrm{~ns}$ for $1.35 \mathrm{~J} / \mathrm{cm}^{2}$ down to $5 \mathrm{~ns}$ for $2.08 \mathrm{~J} / \mathrm{cm}^{2}$ (data not shown here). For fluence levels exceeding $2.67 \mathrm{~J} / \mathrm{cm}^{2}$ [Fig. $5(d)]$ the oscillations are not visible anymore. This can be explained by the temporal period of the reflectivity oscillations which becomes at high fluences too small to be resolved by the streak camera ( $350 \mathrm{ps}$ time resolution under the present conditions).

The RTR measurements finally allow one to determine the distance covered by the ablated layer before the oscillations disappear in the streak camera transients. If $m$ is the number of oscillations observed experimentally, this distance is simply given by $d=m \times \lambda /[2 \operatorname{Re}(n)] \approx m \times \lambda / 2$. This distance decreases for increasing fluences, ranging from more than $4 \mu \mathrm{m}$ for $1.35 \mathrm{~J} / \mathrm{cm}^{2}$ to $\sim 2 \mu \mathrm{m}$ for $2.08 \mathrm{~J} / \mathrm{cm}^{2}$ [Fig. $5(\mathrm{a})$ : $m>16\left(1.35 \mathrm{~J} / \mathrm{cm}^{2}\right)$, Fig. $5(\mathrm{~b}): m<16\left(1.53 \mathrm{~J} / \mathrm{cm}^{2}\right)$, Fig. 5(c): $m<13\left(1.68 \mathrm{~J} / \mathrm{cm}^{2}\right)$, not shown: $\left.m<9\left(2.08 \mathrm{~J} / \mathrm{cm}^{2}\right)\right]$. In all cases, $2 \times d$ is smaller than the coherence length of the laser pulses used in the fs-TRM measurements $(30-40 \mu \mathrm{m})$. Assuming that the observed monotonous decrease of $d$ with laser fluence continues for higher fluences, $d$ should be even smaller in the measurements reported in Fig. 3 for a peak fluence level of $3.10 \mathrm{~J} / \mathrm{cm}^{2}$. The disappearance of the spatial fringes in fs-TRM for high fluences and long delays [10 ns, Fig. 3(f)] can thus be attributed to the modification of the density profile of the ablating layer only (decreasing sharpness of the interfaces, as first proposed in Ref. 13) rather than to the short coherence length of the fs probe pulse.

Along with the shortening of the oscillation period and duration, also the movement of a shoulder (marked with an "S" in streak camera transients) towards longer times can be observed in the entire series [Figs. 5(a)-5(d)]. This shoulder 
arises from the solidification of the liquid layer left on the surface underneath the ablating materials having a higher reflectivity than the solid (see also Sec. III B 2): Since the ablating layer leading to the interference effects is not optically thick, the reflectivity dynamics of the molten layer underneath will contribute to the reflectivity signal. Once this layer undergoes solidification (after some tens of ns) the reflectivity drops significantly which leads to the appearance of the shoulder S (compare to the results presented in Fig. 11). Hence, in this regime of fluences slightly above the ablation threshold, the streak camera is capable of monitoring both the interference effects due to ablating material as well as the solidification dynamics of the material underneath.

The corresponding surface reflectivity transients as measured simultaneously with the photodiode for times up to $1.4 \mu \mathrm{s}$ after the arrival of the pump pulse are shown in Figs. $5(\mathrm{e})-5(\mathrm{~h})$. The rapid reflectivity oscillations as seen in the streak camera transients are not resolved by the photodiode detection system. All transients show a similar behavior and are dominated by an initial drop of the reflectivity followed by slow recovery of the reflectivity on the time scale of a few microseconds. This reflectivity recovery cannot solely be associated with the change of the optical properties of the hot germanium after the resolidification upon cooling down to room temperature, since in $c$-Ge and for our conditions such a process would lead to a reflectivity drop of less than $10 \% .{ }^{9}$ Hence, we rather relate it to the shielding of the probe beam due to thermally induced ablation products arising from thermal evaporation, or the formation of clusters and large particles, which all would absorb and scatter the probe beam radiation. Along with the amplitude of the drop in the reflectivity, this scenario is supported by the characteristic time scale in the nanosecond to microsecond range which has been also observed for the fs-laser pulse ablation of metals. ${ }^{31}$ Note that the final reflectivity change at the end of the measurement interval (after $1.4 \mu \mathrm{s}$ ) is gradually decreasing from $\approx 0$ to $\approx-0.15$, even if a steady state reflectivity value might not have been reached at the highest fluences. This gradual decrease is consistent with the formation of a crater in the surface (always observed in the images of the permanent surface modification), which decreases the reflectivity due to its surface roughness and increasing depth.

\section{B. Fs-laser-induced melting and resolidification}

\section{1. $f s-T R M$}

The evolution of the surface reflectivity has been studied by means of fs-TRM also in the fluence regime between the melting and the ablation thresholds. Figure 6 shows an image sequence for four different peak fluences at a fixed delay of $1 \mathrm{~ns}$. As can be seen, no reflectivity decrease develops in the bright center of the irradiated region [compare to Fig. 3(e)]. None of the images shows features being consistent with the ablation of $\mathrm{Ge}$ in the corresponding fluence interval, suggesting that no ablation of germanium occurs here. Also the final state of the surface does not indicate the formation of an ablation crater on the surface (images not shown).

Surprisingly, one can clearly observe a similar pattern of fringes (like in the regime of ablation) once a certain fluence

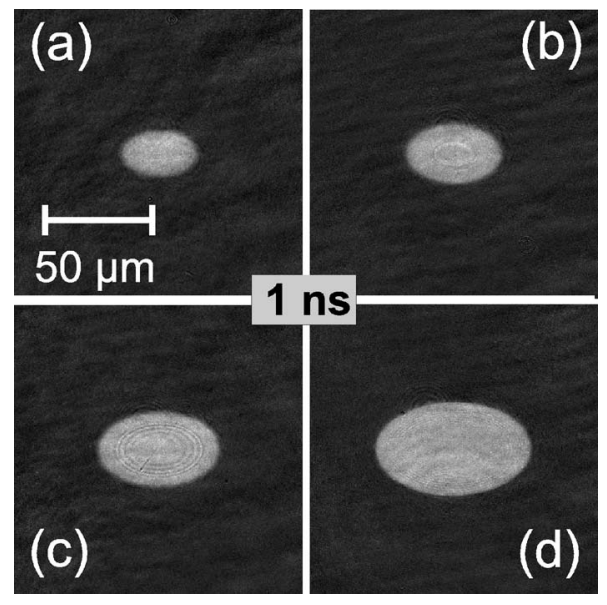

FIG. 6. Images of a germanium surface at $1 \mathrm{~ns}$ delay after the exposure to pump pulse of four different fluences in the melting regime (a): 0.51 , (b): 0.59 , (c): 0.75 , and (d): $1.12 \mathrm{~J} / \mathrm{cm}^{2}$ below the ablation threshold $\left(1.32 \mathrm{~J} / \mathrm{cm}^{2}\right)$. The image sequence is encoded in a linear gray scale with an optimized contrast.

threshold $\left(\sim 0.55 \mathrm{~J} / \mathrm{cm}^{2}\right)$ is exceeded [compare Fig. 6(a) and Figs. 6(b)-6(d)]. These fringes are spatially confined to a central area of the spots which is smaller than the entire molten region for all delay times studied.

In Fig. 7, a direct comparison of representative horizontal reflectivity change profiles is shown. The oscillations in the melting regime show a much smaller degree of reflectivity modulation $\left[\Delta R / R_{f r}(\right.$ melting $) \in[0.50 \cdots 0.85]$, see Fig. $\left.7(\mathrm{a})\right]$ than the previously described fringes in the ablative regime of germanium $\left[\Delta R / R_{f r}\right.$ (ablation) $\in[-1.0 \cdots 1.0]$, see Fig. 7(b)]. This underlines that different processes have to be involved in their formation.

Figure 8 shows a more detailed analysis of the temporal and the spatial reflectivity dynamics in laser-induced melt spots as induced by laser pulses with pump peak fluences of $0.83 \pm 0.03 \mathrm{~J} / \mathrm{cm}^{2}$.

For early times (400 fs delay), a small bright central area without sharp boundary can be seen in Fig. 8(a). Its size increases until the following 10 ps [Figs. 8(b), 1 ps and 8(c), $10 \mathrm{ps}$ ] until a bright spot of $\approx 60 \mu \mathrm{m}$ horizontal diameter with sharp boundaries can be seen after 100 ps [Fig. 8(d)],

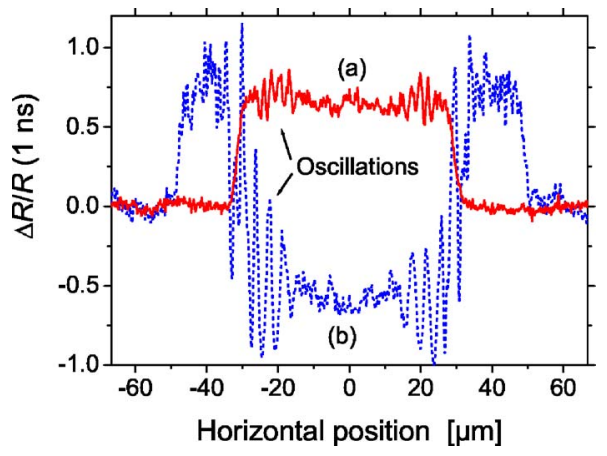

FIG. 7. (Color online) Horizontal profiles of $\Delta R / R$ after $1 \mathrm{~ns}$ delay for two different laser fluence values of (a) $0.83 \mathrm{~J} / \mathrm{cm}^{2}$ [melting, compare Fig. 8(f)] and (b) $3.10 \mathrm{~J} / \mathrm{cm}^{2}$ [ablation, compare Fig. $3(\mathrm{e})]$. 

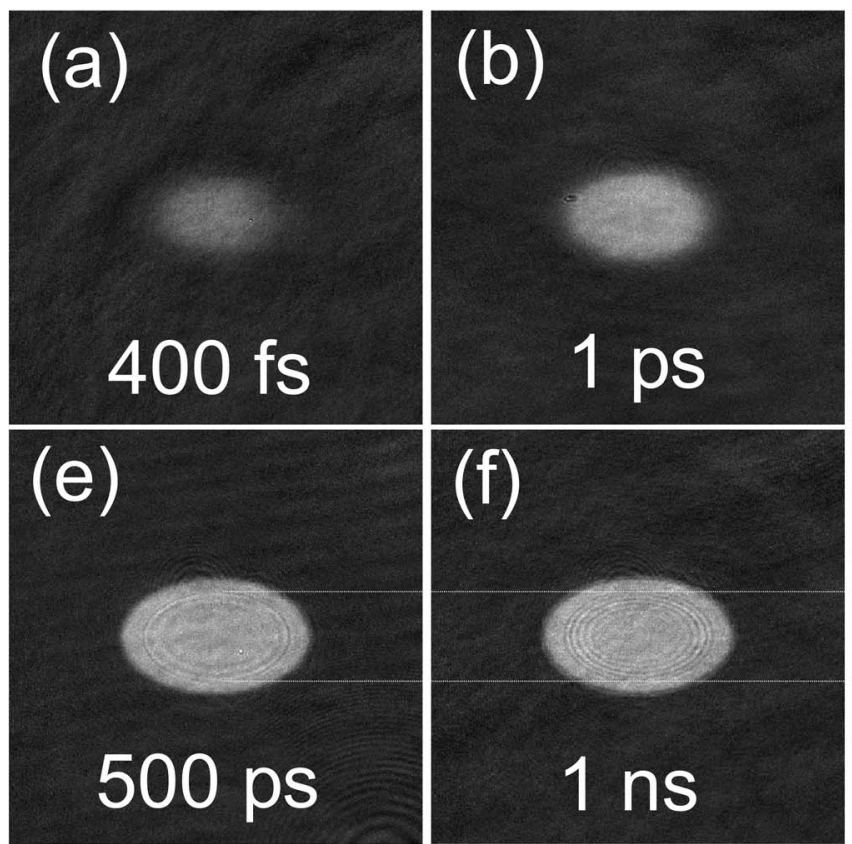
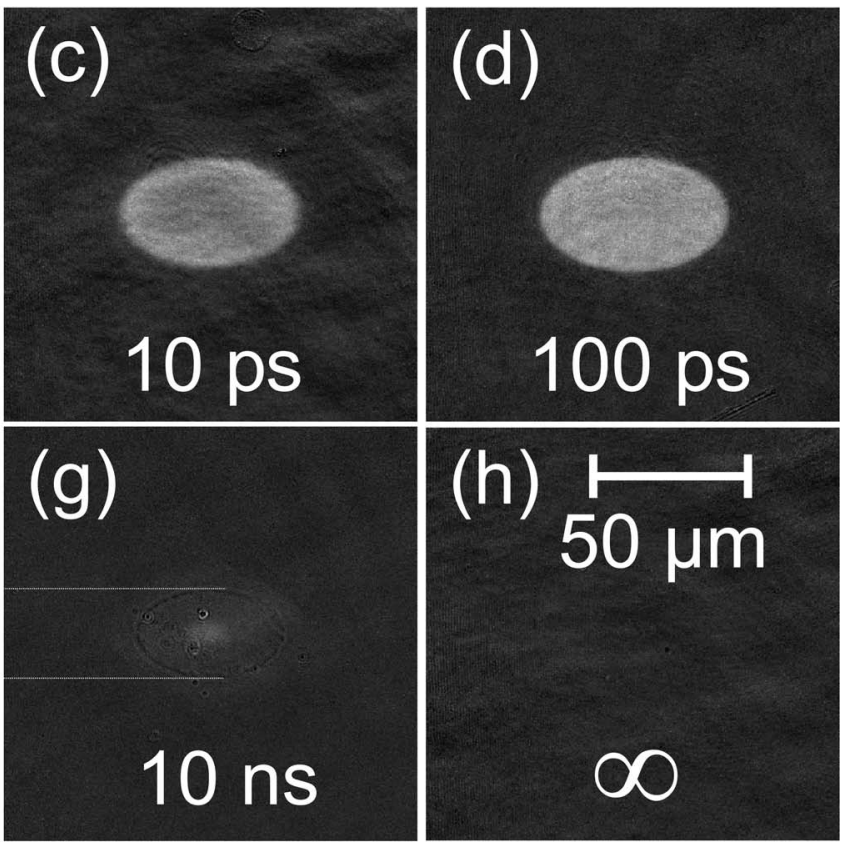

FIG. 8. Images of a germanium surface at different times after the exposure to the pump pulse (pump fluence $0.83 \pm 0.03 \mathrm{~J} / \mathrm{cm}^{2}, \mathrm{melting}$ regime). The image sequence is encoded in a linear gray scale with an optimized contrast.

indicating the formation of a surface layer of molten germanium.

An additional fringe pattern then starts to develop at the surface of the melt pool. As in the case of the fringe pattern observed in the ablative regime, the melt fringes are formed in the center of the spot after $\approx 200$ ps before moving toward the outer edge of the melt pool during the following nanosecond [Figs. 8(e) and 8(f)]. After $10 \mathrm{~ns}$, the surface is nearly completely resolidified and only a very small pool of molten material can be seen in the center [Fig. 8(g)]. Additionally, there is a single fine dark elliptical contour line visible which circumvents the region where the melt fringes have been observed at earlier times. Note that the latter phenomenon is of transient nature only and is not visible anymore in the image of the permanent surface modification [Fig. 8(h)].

Interestingly, the surface reflectivity of the already solidified area is still slightly increased [see Fig. $8(\mathrm{~g}), \Delta R / R$ $\sim 0.02-0.05]$. This increase can be attributed to the change of the optical properties of hot crystalline germanium since at the probe wavelength of $400 \mathrm{~nm}$, the surface reflectivity of $c$-Ge increases by a few percent for rising temperatures close to the melting temperatures [e.g., $\Delta R / R(c-\mathrm{Ge}, 400 \mathrm{~nm}$, $1125 \mathrm{~K}) \sim 0.02-0.03] .^{32}$

In order to reveal the physical origin of the transient fringes in the melt regime, the phase velocity $v_{p}=\omega / k$ $=\sqrt{\frac{\sigma k}{\rho_{l}} \tanh (h k)}$ and the group velocity $v_{g}:=d \omega / d k$ of a surface capillary wave ${ }^{33}$ have been estimated for a melt pool thickness of $h=350 \mathrm{~nm} .{ }^{50}$ Taking the thermophysical constants of liquid germanium (liquid mass density $\rho_{l}=5.5$ $\times 10^{3} \mathrm{~kg} / \mathrm{m}^{3}$, surface tension $\left.\sigma=600 \times 10^{3} \mathrm{~N} / \mathrm{m}\right)^{34}$ and an experimentally observed fringe spacing of $\Lambda=2 \pi / k$ $=2.42 \mu \mathrm{m}$, values of $v_{p}=14.3 \mathrm{~m} / \mathrm{s}$ and $v_{g}=25.8 \mathrm{~m} / \mathrm{s}$ are found. ${ }^{51}$ Both values are by three orders of magnitude smaller than the speed of the fringes moving outwards to the edge of the spot $(25 \mathrm{~km} / \mathrm{s}) .{ }^{52}$
The striking similarity in the temporal development (100 ps-few ns) and in the spatial period of the fringes (few micrometers) suggests that the low-contrast fringes in the melting regime are generated by the selective ablation of the native oxide layer which is covering the Ge sample surface. This effect of surface oxide removal upon fs-laser pulse irradiation at fluence levels between the melting and the ablation threshold has already been observed for silicon in ex situ optical and atomic force microscopic studies, ${ }^{35}$ whereas here we provide direct evidence for such a process by an optical in situ technique. A selective oxide removal has also been reported for ns-laser pulse irradiation of germanium wafers with fluences below their ablation threshold at $193 \mathrm{~nm}$ wavelength where the oxide is slightly absorbent. ${ }^{8}$ Furthermore, the hypothesis of oxide removal is fully consistent with our experimental observations, i.e., (i) the existence of a threshold behavior for the appearance of the fringes, (ii) the spatial confinement of the fringes to a region smaller than the extent of melting, and (iii) the thermophysical properties of Ge and the covering oxide layer. We will discuss these issues in more details in the following:

Ad (i) and (ii): For a fixed delay of $1 \mathrm{~ns}$, we have analyzed the outer horizontal diameters of the molten region as well as those of the fringe pattern as a function of the peak laser fluence $\Phi_{0}$ (Fig. 9). In both cases, a functional dependence of $D^{2}=2 w_{0}^{2} \ln \left(\Phi_{0} / \Phi_{t h}\right)$ is found $\left(w_{0}=50 \mu \mathrm{m}\right.$ : Gaussian $1 / e^{2}$-beam radius). This dependence is exactly the one predicted for any threshold $\left(\Phi_{t h}\right)$ dependent process induced by a spatially Gaussian pulse. Moreover, back-extrapolating $D^{2} \rightarrow 0$ allows a quantitative determination of both thresholds. A value of $\Phi_{t h, f r}=0.55 \mathrm{~J} / \mathrm{cm}^{2}$ has been found for the onset of the appearance of the fringes, whereas for melting we have found the smaller value of $\Phi_{m}=0.39 \mathrm{~J} / \mathrm{cm}^{2}$. Since the threshold for fringes is higher than the melting threshold, this explains also why the fringes are confined to an area smaller than the molten region. 


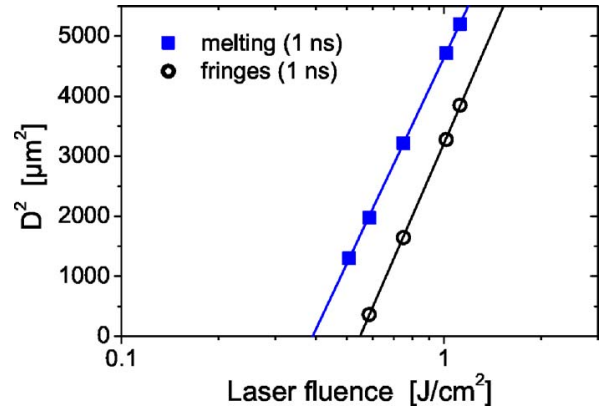

FIG. 9. (Color online) Square of the transient horizontal diameters $D^{2}$ at a pump-probe delay of $1 \mathrm{~ns}$ as a function of the incident laser fluence $\Phi_{0}$ for fluence below the ablation threshold of $c$-Ge. The full squares show the data for the molten region, whereas the open circles represent the data for the transient fringe pattern. The solid line indicate least-squares-fits for irradiation with a spatially Gaussian laser beam.

Ad (iii): The hypothesis is further supported by the thermophysical data of germanium and its native oxide. Amorphous germanium dioxide $\left(a-\mathrm{GeO}_{2}\right)$ melts at higher temperatures than germanium $\left[T_{m}\left(a-\mathrm{GeO}_{2}\right)=1347 \mathrm{~K}>T_{m}(c-\mathrm{Ge})\right.$ $=1210 \mathrm{~K}]^{30}$ but it evaporates at a temperature $\approx 500^{\circ}$ lower than germanium does $\left[T_{b}\left(a-\mathrm{GeO}_{2}\right)=2625 \mathrm{~K}<T_{b}(c-\mathrm{Ge})\right.$ $=3104 \mathrm{~K}] \cdot{ }^{30}$ Since the laser-induced temperature rise is monotonously increasing related to the local laser fluence, a thermally induced oxide removal will occur in the melting regime of germanium at fluences lower than the ablation threshold.

It should be noted here that for thermally grown oxide films (50-1200 nm thickness) on silicon, a blistering and delamination of the oxide films has been observed very recently by post-irradiation atomic force and optical microscopy following NIR fs-laser pulse irradiation. ${ }^{36}$ This also suggests that in our case of a thin native oxide layer on germanium its removal should start at the semiconductor/ oxide interface.

In Fig. 10 both the horizontal diameter of the molten region and the normalized surface reflectivity change in the center of the irradiated area are plotted as a function of the delay time between the pump and the probe pulse.

The horizontal diameter of the molten surface region increases during the first $10 \mathrm{ps}$ until a saturation value of $60 \mu \mathrm{m}$ is reached [Fig. 10(a)]. This might be indicative of a thermal melting process. Between $1 \mathrm{~ns}$ and $10 \mathrm{~ns}$ the diameter starts to decrease again upon solidification of the melt pool which is completed for times between 10 and $100 \mathrm{~ns}$.

In contrast, the normalized surface reflectivity change increases within the first picosecond to the experimentally measured level of the liquid $[\Delta R / R(\exp ) \sim 0.65]$ at $400 \mathrm{~nm}$ probe wavelength [Fig. 10(a)]. For times between 1 ps and approximately $100 \mathrm{ps}$, a transient reflectivity decrease up to $25 \%$ can be seen. Since this change is too large to be explained only by the changes of the optical properties of a superheated liquid [as it has been observed in other semiconductors like silicon ${ }^{26}$ and GaAs (Ref. 17)], the onset of ablation of the oxide layer may additionally contribute to it. After 100 ps delay time, the liquid reflectivity value is recov-
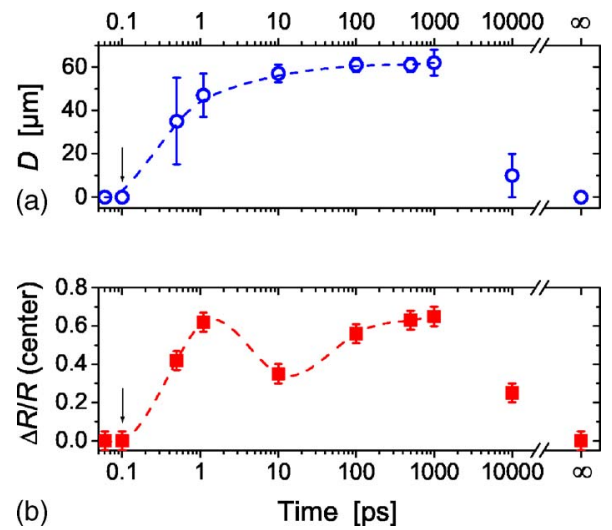

FIG. 10. (Color online) Horizontal diameter $D$ of the molten region (a) and normalized surface reflectivity change $\Delta R / R$ in center (b) as a function of time as measured for the irradiated regions shown in Fig. 8. Note the logarithmic time axis. The true zero delay is marked by an arrow. The final reflectivity change $(\Delta t=\infty)$ is marked on the right-hand horizontal axis. The lines are to guide the eye.

ered again. No permanent surface reflectivity change has been observed after the resolidification $[\Delta R / R(\infty)=0]$.

\section{Ps- and ns-RTR}

As in the ablative regime, the fs-laser induced melting has been studied on longer timescales (ns- $\mu \mathrm{s}$ ) using the RTRsetup. Figure 11 shows the temporal evolution of the normalized surface reflectivity change as probed simultaneously by the streak camera $[(\mathrm{a})-(\mathrm{c})]$ and the photodiode $[(\mathrm{d})-(\mathrm{f})]$ in

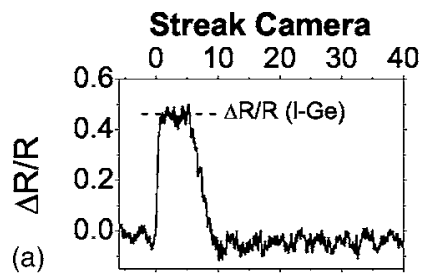

(d)
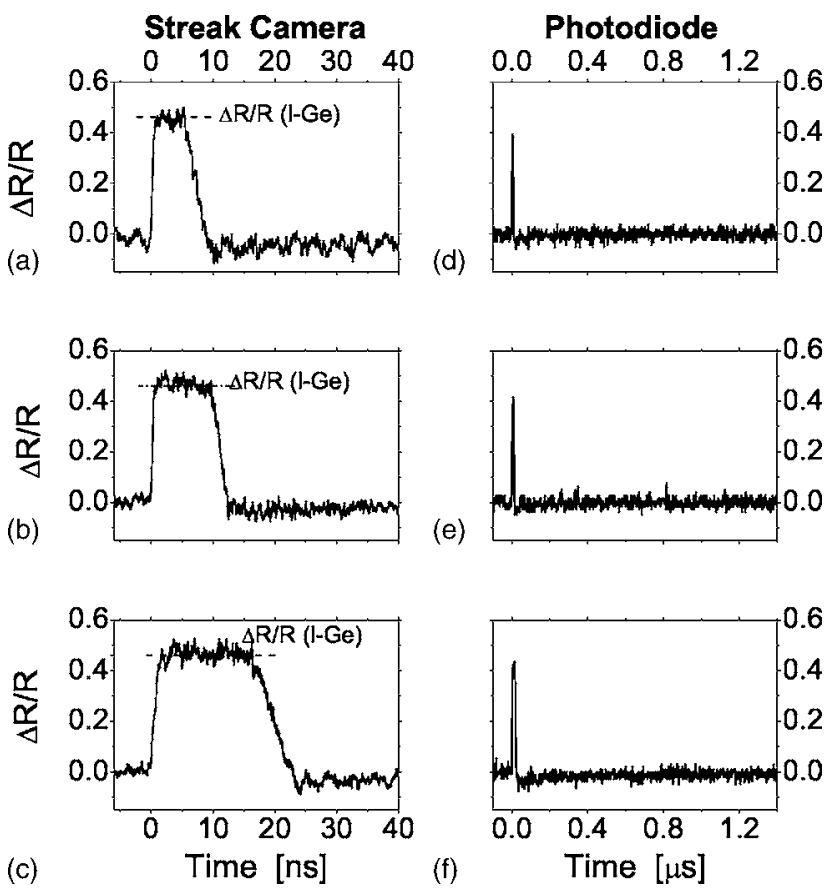

FIG. 11. Normalized surface reflectivity change as a function of time as measured with a streak camera (a)-(c) and with a photodiode (d)-(f) at three different fluence levels $\left[(\mathrm{a})+(\mathrm{d}): 0.75 \mathrm{~J} / \mathrm{cm}^{2}\right.$, (b) $\left.+(\mathrm{e}): 0.84 \mathrm{~J} / \mathrm{cm}^{2},(\mathrm{c})+(\mathrm{f}): 1.32 \mathrm{~J} / \mathrm{cm}^{2}\right]$. 
TABLE I. Optical constants (complex refractive index $n+i k$ ) used for the thin film optical calculations.

\begin{tabular}{llcccc}
\hline \hline Phase & $\begin{array}{l}\text { Wavelength } \\
\lambda\end{array}$ & $\begin{array}{c}\text { Refractive index } \\
(n+i k)\end{array}$ & $\begin{array}{c}\text { Opt. penetration } \\
\text { depth } \lambda /(4 \pi k)\end{array}$ & Ref. \\
& {$[\mathrm{nm}]$} & $n$ & $k$ & {$[\mathrm{~nm}]$} & \\
\hline$c-\mathrm{Ge}$ & 400 & 4.14 & 2.21 & 14.4 & 20 \\
& 514.5 & 4.61 & 2.45 & 16.7 & 20 \\
$\ell-\mathrm{Ge}$ & 400 & 1.81 & 4.25 & 7.5 & 25 \\
& 514.5 & 2.51 & 5.12 & 8.0 & 25 \\
$a-\mathrm{Ge}$ & 400 & 3.48 & 2.89 & 11.0 & 42 \\
& 514.5 & 4.30 & 2.30 & 17.8 & 42 \\
$a-\mathrm{GeO}_{2}$ & 400 & 1.64 & 0.00 & $\infty$ & 43 \\
& 514.5 & 1.61 & 0.00 & $\infty$ & 43 \\
\hline \hline
\end{tabular}

the center of the irradiated region upon irradiation at three different pump peak fluences in the melting regime.

In all streak camera transients [Figs. 11(a)-11(c)], a similar behavior can be seen, i.e., a rapid reflectivity increase to a reflectivity plateau with a saturated level of $(\Delta R / R)_{\max }$ $\approx 0.46$. This is indicative of the formation of a molten layer at the surface with a thickness greater than the optical penetration depth of the probing $514.5 \mathrm{~nm}$ radiation $(8 \mathrm{~nm}$, see Table I). The experimentally observed plateau values between 0.45 and 0.48 in the normalized reflectivity change perfectly agree with the value of 0.46 obtained by thin film optical simulations for an optically thick layer of molten germanium, which is covered by a native oxide layer (see Sec. III B 3), as indicated by dashed horizontal lines (Fig. 11). The plateau lasts only for up to a few tens of nanoseconds (fluence dependent). After the plateau, the reflectivity change decreases again to a final level around $\Delta R / R(40 \mathrm{~ns}) \sim$ -0.04 . Depending on the fluence, this occurs within $3 \mathrm{~ns}$ [Fig. 11(b)] and $8 \mathrm{~ns}$ [Fig. 11(c)]. Analyzing the temporal onset of resolidification in the melting regime shows that the melt-duration scales approximately linearly with the laser fluence (data not shown here). Along with the high thermal conductivity of the crystalline material $\left[0.6 \mathrm{~W} /(\mathrm{cm} \cdot \mathrm{K})^{2}\right]$ this is indicative of an interfacial resolidification process, which will be analyzed in detail in Sec. III B 3.

Since the complementary photodiode transients [Figs. 11(d)-11(f)] do not fully resolve the reflectivity plateau, their maximum values $(\Delta R / R)_{\max }$ are systematically below that measured by the streak camera. It is interesting to note in this context that immediately after solidification the reflectivity level is slightly lower than the initial one [especially in Figs. 11(c) and 11(f)]. This can be explained by the temperature dependence of the optical properties of $c$-Ge. ${ }^{9}$ However, it can be seen at longer times that the reflectivity recovers its initial value, i.e., $\Delta R / R(1.4 \mu \mathrm{s})=0$.

Note that the fluence for the reflectivity transient shown in Figs. 11(b) and $11(\mathrm{e})\left[0.84 \mathrm{~J} / \mathrm{cm}^{2}\right]$ is comparable to the one of the image series depicted in Fig. $8\left[0.83 \mathrm{~J} / \mathrm{cm}^{2}\right]$. Consistently, in both figures the resolidification of the liquid layer can be seen to occur after $\approx 10$ ns. However, due to the small modulation depth of the reflectivity oscillations when compared to the signal-to-noise ratio, the fringes in the melting regime cannot be resolved in our RTR measurements, as opposed to the ablative regime in which this was possible (Fig. 5).

\section{Calculations of the reflectivity changes}

Additional insights into the processes occurring in the melting regime can be obtained from thin film optical calculations, especially by modeling the surface reflectivity change upon melting and resolidification, which might induce the formation of an amorphous top layer. For bulk semiconductors it was found that the resolidification of a laser-induced melt layer occurs interfacially from the solid/ liquid interface and the solidifying material turns to an amorphous state when a critical speed in the order of $1-25 \mathrm{~m} / \mathrm{s}$ is exceeded for the velocity of the resolidification front. ${ }^{37-40} \mathrm{At}$ such velocities, there is not enough time for the nucleation of a crystalline phase, which leads to the formation of the amorphous material.

The evolution of the reflectivity in such a scenario has been modeled by considering a thin film of molten or amorphous material with variable thickness on top of a crystalline substrate. Additionally, it is assumed that the surface exposed to air is covered by a transparent native layer of amorphous germanium dioxide $\left(a-\mathrm{GeO}_{2}\right)$. The optical model used takes into consideration the fully coherent superposition of Fresnel reflections at all interfaces [air/oxide, oxide/ liquid(amorphous) and liquid(amorphous)/solid] for the given probe wavelengths $(400 \mathrm{~nm}$ or $514.5 \mathrm{~nm})$, both at normal incidence. ${ }^{35,41}$ For direct comparison, also the case of an oxide-free surface has been considered. The optical constants $n+i k$ for the different material phases $(c-\mathrm{Ge}, a-\mathrm{Ge}, \ell-\mathrm{Ge}$, and $\left.a-\mathrm{GeO}_{2}\right)$ used for the thin film optical calculations are summarized in Table I.

In our calculations, the presence of few nanometers thick transparent oxide layer on the surface causes the reflectivities to be lowered by a few percent. For example, the reflectivity of bulk single-crystalline germanium at both probe wavelengths are $R(c-\mathrm{Ge}, 400 \mathrm{~nm})=0.47$ and $R(c-\mathrm{Ge}, 514.5 \mathrm{~nm})$ $=0.51$, whereas the values for native oxide covered bulk material are $R\left(a-\mathrm{GeO}_{2}(3.0 \mathrm{~nm}) / c-\mathrm{Ge}, 400 \mathrm{~nm}\right)=0.46$ and $R\left(a-\mathrm{GeO}_{2}(3.0 \mathrm{~nm}) / c-\mathrm{Ge}, 514.5 \mathrm{~nm}\right)=0.50$, respectively.

Since the native oxide layer is already present on the nonirradiated wafer surface, the latter values have been used to evaluate all normalized reflectivity changes in our thin film optical calculations. Then, the removal of the native oxide layer causes an increase of the normalized surface reflectivity change-in contrast to heating effects, which are lowering the reflectivity by up to $10 \%$ (see above). However, these calculations indicate that the differences in the reflectivity between an oxide-covered and an oxide-free wafer surface are too small to be detected directly by our optical probing setups. Nevertheless, following our hypothesis of oxide removal, its direct experimental detection seems to be possible when additional interference phenomena are involved as it is the case in Fig. 8, giving rise to the fringe pattern.

(a) Melt-layer: The results of these surface reflectivity calculations at both probe wavelengths are shown in Fig. 12, where the normalized surface reflectivity change $\Delta R / R$ is evaluated for different thicknesses $d_{l}$ of a liquid layer of Ge 


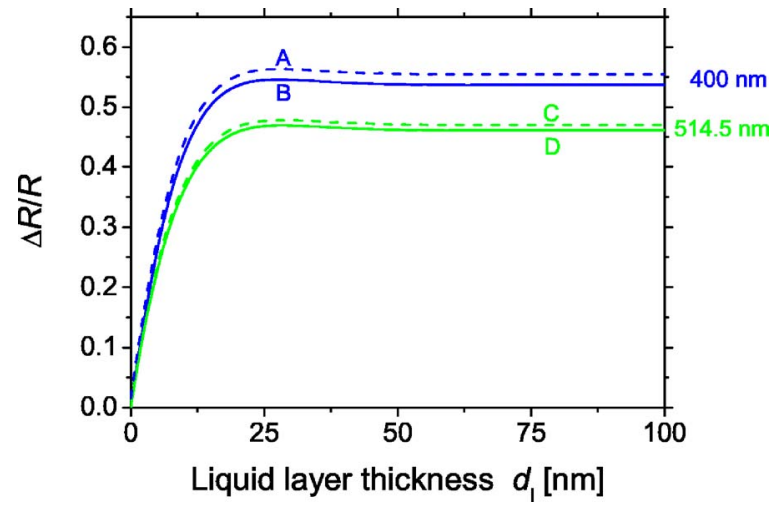

FIG. 12. (Color online) Normalized surface reflectivity change obtained by thin film optical calculations $\left(0^{\circ}\right.$ angle of incidence) of a layer of liquid Ge with variable thickness $d_{l}$ on top of a singlecrystalline substrate at two different probe laser wavelengths (400 $\mathrm{nm}$ and $514.5 \mathrm{~nm}$ ). For the solid curves, an additional $3 \mathrm{~nm}$ thick native oxide layer is considered, whereas the dashed curves represent calculations without the oxide layer. The optical constants used are listed in Table I. For labeling of the curves refer to the text.

on top of the crystalline substrate. The reflectivity change has been calculated for a layer system with a $3.0 \mathrm{~nm}$ thick native oxide layer (solid lines) and also without any covering oxide (dashed lines).

In all curves (Fig. 12, curves A-D), a rapid increase of the surface reflectivity with increasing liquid layer thickness can be observed before reaching a saturation level once the liquid layer becomes optically thick. At the probe wavelength of $400 \mathrm{~nm}$ (Fig. 12, curves A and B), we do not observe a satisfying agreement between the experimentally determined [compare Figs. 7(a) and 10(b)] and the calculated reflectivity changes for the liquid phase since the average values in $\Delta R / R$ are too high by as much as $15 \%$ for the experimental data (compare Table II). The origin is not fully clear at the moment, but the deviation is significantly larger than the experimental error. Some uncertainty in the calculated values might arise from the optical constants used for $\ell$-Ge which were extrapolated to this probe wavelength from a leastsquares-fit based on a Drude model. ${ }^{25}$ At $514.5 \mathrm{~nm}$ probe wavelength, both saturation levels of the normalized reflectivity change for the oxide-free liquid (Fig. 12, curve C, $\Delta R / R_{\text {sat }}=0.47$ ) and the oxide-covered liquid (Fig. 12, curve $\left.\mathrm{D}, \Delta R / R_{\text {sat }}=0.46\right)$ are in excellent agreement with the experimental data shown in Fig. $11(\Delta R / R=0.45-0.48)$.

Along with the experimental data shown in Fig. 11, the thin film optical calculations allow a quantitative estimation of the velocity of the resolidification front while approaching the surface: it is seen in Fig. 12 (curves C and D) that the normalized reflectivity starts to decrease rapidly below the bulk reflectivity level of the liquid at a thickness of $\sim 20 \mathrm{~nm}$. Accordingly we can use the transients in Fig. 11 to estimate the time taken by the solidification front to travel a distance equivalent to this liquid layer thickness. This time corresponds to the time elapsed between the end of the reflectivity plateau and the time at which the reflectivity reaches the value of hot solid germanium which ranges from 3 to $8 \mathrm{~ns}$. Hence, mean interfacial speeds between 2.5 and $7 \mathrm{~m} / \mathrm{s}$ are estimated.
TABLE II. Surface reflectivities obtained by thin film optical calculations at different probe wavelengths (400 nm, $514.5 \mathrm{~nm})$ and for normal incident radiation. Additionally, the corresponding normalized reflectivity changes $\Delta R / R=\left(R-R_{c}\right) / R_{c}$ are listed, where $R_{c}$ represents the reflectivity of $c$-Ge covered by a $3.0 \mathrm{~nm}$ thick native layer of $a-\mathrm{GeO}_{2}$. For comparison, the experimentally obtained values are listed. n.e.: no evidence.

\begin{tabular}{rlccc}
\hline \hline Layer system & $\begin{array}{l}\text { Wave- } \\
\text { length } \\
\lambda[\mathrm{nm}]\end{array}$ & $\begin{array}{c}\text { (Calc. }) \\
R\end{array}$ & $\begin{array}{c}\text { Reflectivity } \\
(\text { Calc. })\end{array}$ & $\begin{array}{c}\Delta R / R \\
(\text { Exp. }) \\
\Delta R / R\end{array}$ \\
\hline$a-\mathrm{GeO}_{2}(3.0 \mathrm{~nm}) / c$-Ge & 400 & 0.464 & 0 & 0 \\
& 514.5 & 0.503 & 0 & 0 \\
$c$-Ge & 400 & 0.471 & +0.02 & - \\
& 514.5 & 0.508 & +0.01 & - \\
$a-\mathrm{GeO}_{2}(3.0 \mathrm{~nm}) / \ell-\mathrm{Ge}$ & 400 & 0.713 & +0.54 & $0.50-0.85$ \\
& 514.5 & 0.735 & +0.46 & $0.45-0.48$ \\
$\ell-\mathrm{Ge}$ & 400 & 0.721 & +0.55 & $0.50-0.85$ \\
& 514.5 & 0.739 & +0.47 & $0.45-0.48$ \\
$a-\mathrm{GeO}_{2}(3.0 \mathrm{~nm}) / a-\mathrm{Ge}$ & 400 & 0.511 & +0.08 & n.e. \\
& 514.5 & 0.480 & -0.05 & n.e. \\
$a-\mathrm{Ge}$ & 400 & 0.510 & +0.10 & n.e. \\
& 514.5 & 0.485 & -0.04 & n.e. \\
\hline \hline
\end{tabular}

(b) Amorphous-layer: This speed is lower than the critical speed of resolidification in other elemental semiconductors with a diamond structure, such as silicon, where values between 12 and $25 \mathrm{~m} / \mathrm{s}$ (depending on the crystal orientation) have to be exceeded for inducing amorphization. ${ }^{37,38}$

Unfortunately, and to the best of our knowledge, no experimental data are available for the critical speed of resolidification in germanium. However, Spaepen and Turnbull have estimated the maximum crystallization speed for germanium to be approx. $100 \mathrm{~m} / \mathrm{s}$ (Refs. 44 and 45).

In order to investigate the possible existence of an amorphus top layer induced by fs-laser pulse irradiation, additional thin film optical calculations have been performed for a thin layer of amorphous material $(a-\mathrm{Ge})$ with variable thickness $d_{a}$ on the single-crystalline wafer $(c-\mathrm{Ge})$. The results at both probe wavelengths are shown in Fig. 13. In analogy to the case of a thin liquid layer, the normalized surface reflectivity change has been calculated for a layer system with a $3.0 \mathrm{~nm}$ thick native oxide layer (solid lines) and also without any covering oxide (dashed lines).

At $400 \mathrm{~nm}$ wavelength, both curves A and B show a rapid increase of the surface reflectivity with increasing layer thickness, followed by a damped oscillation before reaching the saturation value, i.e., the "bulk" reflectivity of amorphous germanium. The oscillation is caused by constructive and destructive interference effects due to the partial reflections mainly at the $a-\mathrm{GeO}_{2} / \ell-\mathrm{Ge}(a-\mathrm{Ge})$ and the $\ell-\mathrm{Ge}(a-\mathrm{Ge}) /$ $c$-Ge interfaces. The extinction coefficient $k$ determines how strong the oscillation is damped. For $a$-Ge films, thicknesses larger than $75 \mathrm{~nm}$ are needed to saturate the reflectivity. These saturation values are $\Delta R / R_{\text {sat }}=0.10$ for the oxide free (Fig. 13, curve A) and $\Delta R / R_{\text {sat }}=0.08$ for the native oxidecovered $a$-Ge surface (Fig. 13, curve B). 


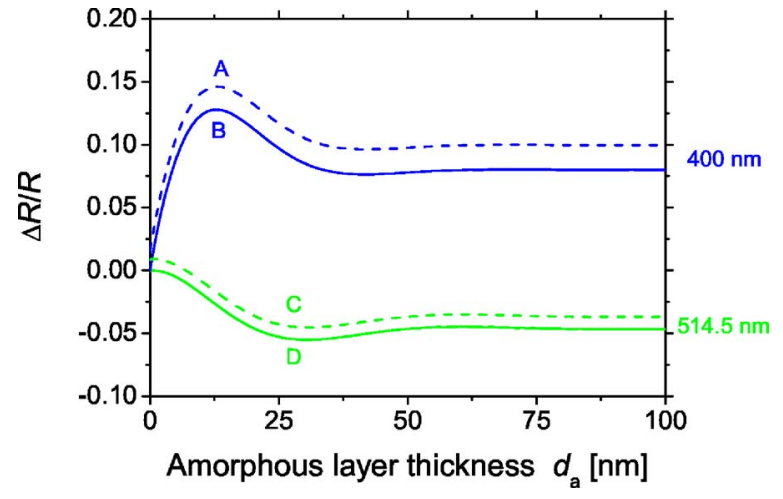

FIG. 13. (Color online) Normalized surface reflectivity change obtained by thin film optical simulations $\left(0^{\circ}\right.$ angle of incidence) of a layer of amorphous Ge with variable thickness $d_{a}$ on top of a single-crystalline substrate at two different probe laser wavelengths (400 $\mathrm{nm}$ and $514.5 \mathrm{~nm}$ ). For the solid curves, an additional $3 \mathrm{~nm}$ thick native oxide layer is considered, whereas the dashed curves represent calculations without the oxide layer. The optical constants used are listed in Table I. For labeling of the curves refer to the text.

At $514.5 \mathrm{~nm}$ wavelength, the normalized reflectivity change first decreases before reaching a small negative saturation level of some percent (see Fig. 13, curves C and D, and the quantitative values given in Table II).

However, given the absence of any observable permanent change in the surface reflectivity at both probe wavelengths (400 $\mathrm{nm}$ and $514.5 \mathrm{~nm}$ ) after fs-laser pulse irradiation at fluences below the ablation threshold, we conclude that there is no evidence for the formation of a superficial amorphous top layer from the melt. Apparently, and in contrast to the femtosecond laser pulse irradiation of other semiconductors like (111)-silicon ${ }^{35}$ or (100)-indium phosphide ${ }^{40,46}$ under similar conditions, the regrowth velocity of the liquid-solid interface is sufficiently low to prevent amorphization of the surface. Hence, our values of 2.5 to $7.0 \mathrm{~m} / \mathrm{s}$ for the interfacial regrowth speed at the end of the solidification process represent a lower limit for the critical resolidification speed in (100)-germanium: despite the fact that the velocity of the liquid/solid interface speeds up at the end of the solidification process driven by the gradually increasing supercooling of the melt, ${ }^{47}$ no amorphization has been observed in the present case. Hence, the maximum interface velocity reached here upon the fs-laser pulse induced solidification of germanium has to be smaller than the critical velocity for amorphization to occur.

Two additional aspects have to be mentioned with respect to the different behavior of (100)-Ge upon fs-laser induced irradiation when compared to the other mentioned semiconductors. First, it has been reported by Cullis et al. for ns-laser irradiation of various semiconductors ( $\mathrm{Si}, \mathrm{Ge}, \mathrm{GaAs}, \mathrm{GaP}$ ) that the crystal orientation significantly influences the resolidification behavior of the material. ${ }^{38,48}$ Crystals with (111) orientation generally exhibit lower recrystallization velocities with consequential enhancement of melt undercooling and a higher amorphous phase nucleation probability when compared to (100)-oriented samples. ${ }^{48}$ In other words, (111) crystals amorphize more easily than (100) oriented ones.

Secondly, the recrystallization behavior is directly linked to the heat conduction within the material. Materials with lower thermal diffusivity values reduce the undercooling of the melt (which determines the speed of the solidification front) and therefore slow down the recrystallization rate. Hence, it is less likely that the critical interface speed for crystallization is exceeded in these materials. This trend is also seen in the magnitudes of the thermal diffusivity of $c$-Si $\quad\left(0.94 \mathrm{~cm}^{2} / \mathrm{s}\right),{ }^{2} \quad c$-InP $\quad\left(0.47 \mathrm{~cm}^{2} / \mathrm{s}\right),{ }^{2}$ and $c$-Ge $\left(0.35 \mathrm{~cm}^{2} / \mathrm{s}\right),{ }^{2}$ which consequently reflect the tendency to form an amorphous top layer at the end of the solidification process (for a given crystal orientation).

\section{CONCLUSIONS}

In summary, we have studied the temporal dynamics of melting, ablation, and resolidification upon titanium:sapphire femtosecond laser pulse irradiation of single-crystalline germanium. Femtosecond time-resolved microscopic imaging and complementary streak camera and photodiode measurements allowed a complete characterization of the phase change dynamics in a time span covering more than seven orders of magnitude ( $\sim 100$ fs up to $1.4 \mu$ s). Our results confirm the few previous studies on ultrafast phase transitions in germanium and provide additional insights due to the joint application of fs-time-resolved and real-time probing techniques: Evidence is presented that the native oxide layer present on germanium wafers can be removed selectively at fluences above the melting but below the ablation threshold of germanium, leading to a transient surface reflectivity pattern in the picosecond to nanosecond range, without a measurable change of the permanent surface reflectivity. In contrast to the femtosecond laser pulse irradiation of other semiconductors, no indication for a laser-induced permanent superficial amorphization was found. From these observation along with the transient solidification behavior of the molten germanium on a time scale of some nanoseconds, a lower limit for the critical speed of the liquid/solid interface between 2.5 and $7.0 \mathrm{~m} / \mathrm{s}$ has been deduced for the fs-laser irradiation of (100) Ge. For single laser pulses with fluences slightly exceeding the ablation threshold, we have been able to observe reflectivity oscillations (caused by the transient Newton-fringe pattern observed in femtosecond microscopy) up to some tens of nanoseconds by means of real-time probing with a streak camera. This extends the maximum duration over which this phenomenon has been monitored via interference phenomena using femtosecond time resolved microscopy by one order of magnitude.

\section{ACKNOWLEDGMENTS}

This work has been partially supported by the TEC 200500074 project and by the EU in the frame of the TMR project FLASH (MRTN-CT-2003-503641). One author (G.B.) acknowledges the funding in the frame of the same project. Another author (J.B.) acknowledges the funding of the C.S.I.C. through a contract in the frame of the I3P Programme (Ref. I3P-PC2002), co-funded by the European Social Fund. The authors would like to thank R. Serna for performing the ellipsometry measurements. 
*Corresponding author, electronic address: bonse@mbi-berlin.de; Present address: Max-Born-Institut für Nichtlineare Optik und Kurzzeitspektroskopie, Max-Born-Strasse 2A, D-12489 Berlin, Germany.

'Present address: Laboratoire de Spectrométrie Ionique et Moléculaire, UMR CNRS 5579, Université Claude Bernard-Lyon I, Bâtiment Alfred Kastler, 43 Boulevard du 11 Novembre 1918, F-69622 Villeurbanne Cedex, France.

Electronic address: j.solis@io.cfmac.csic.es

${ }^{1}$ Wikipedia Encyclopedia, http://en.wikipedia.org/wiki/ Germanium

${ }^{2}$ A. Dargys and J. Kundrotas, Handbook on Physical Properties of Ge, Si, GaAs and InP (Science and Encyclopedia Publishers, Vilnius, 1994).

${ }^{3}$ A. Elci, M. O. Scully, A. L. Smirl, and J. C. Matter, Phys. Rev. B 16, 191 (1977).

${ }^{4}$ G. E. Jellison, D. H. Lowndes, D. N. Mashburn, and R. F. Wood, Phys. Rev. B 34, 2407 (1986).

${ }^{5}$ A. Othonos, H. M. van Driel, J. F. Young, and P. J. Kelly, Phys. Rev. B 43, 6682 (1991).

${ }^{6}$ W. R. Sooy, M. Geller, and D. P. Bortfeld, Appl. Phys. Lett. 5, 54 (1964).

${ }^{7}$ D. H. Auston, J. A. Golovchenko, A. L. Simons, and T. N. C. Venkatesan, Appl. Phys. Lett. 34, 777 (1979).

${ }^{8}$ J. Solis, F. Vega, and C. N. Afonso, Appl. Phys. A: Mater. Sci. Process. 62, 197 (1996).

${ }^{9}$ N. Chaoui, J. Siegel, J. Solis, and C. N. Afonso, J. Appl. Phys. 89, 3763 (2001).

${ }^{10}$ N. Chaoui, J. Siegel, S. M. Wiggins, and J. Solis, Appl. Phys. Lett. 86, 221901 (2005).

${ }^{11}$ K. Sokolowski-Tinten, A. Cavalleri, and D. von der Linde, Appl. Phys. A: Mater. Sci. Process. 69, 577 (1999).

${ }^{12}$ C. W. Siders, A. Cavalleri, K. Sokolowski-Tinten, C. Toth, T. Guo, M. Kammler, M. H. von Hoegen, K. R. Wilson, D. von der Linde, and C. P. J. Barty, Science 286, 1340 (1999).

${ }^{13}$ B. Rethfeld, V. V. Temnov, K. Sokolowski-Tinten, S. I. Anisimov, and D. von der Linde, Proc. SPIE 4760, 72 (2002).

${ }^{14}$ K. Sokolowski-Tinten, S. Kudryashov, V. Temnov, J. Bialkowski, M. Boing, D. von der Linde, A. Cavalleri, H. O. Jeschke, M. E. Garcia, and K. H. Bennemann, Ultrafast Phenomena XII, Springer Series in Chemical Physics 66 (Springer-Verlag, Heidelberg, 2000).

${ }^{15}$ M. C. Downer, R. L. Fork, and C. V. Shank, J. Opt. Soc. Am. B 2, 595 (1985).

${ }^{16}$ D. von der Linde and K. Sokolowski-Tinten, Appl. Surf. Sci. 154-155, 1 (2000).

${ }^{17}$ K. Sokolowski-Tinten, J. Bialkowski, M. Boing, A. Cavalleri, and D. von der Linde, Phys. Rev. B 58, R11805 (1998).

${ }^{18}$ V. V. Temnov, Ph.D. thesis, University of Duisburg-Essen, Germany, 2004.

${ }^{19}$ J. Solis, J. Siegel, and C. N. Afonso, Rev. Sci. Instrum. 71, 1595 (2000).

${ }^{20}$ Handbook of Optical Constants of Solids, edited by E. D. Palik (Academic Press, Orlando, Florida, 1998).

${ }^{21}$ P. Stampfli and K. H. Bennemann, Phys. Rev. B 42, 7163 (1990).

${ }^{22}$ C. V. Shank, R. Yen, and C. Hirlimann, Phys. Rev. Lett. 50, 454 (1983).
${ }^{23}$ I. L. Shumay and U. Höfer, Phys. Rev. B 53, 15878 (1996).

${ }^{24}$ J. Bonse, S. M. Wiggins, and J. Solis, J. Appl. Phys. 96, 2628 (2004).

${ }^{25}$ G. E. Jellison and D. H. Lowndes, Appl. Phys. Lett. 51, 352 (1987).

${ }^{26}$ J. Boneberg, O. Yavas, B. Mierswa, and P. Leiderer, Phys. Status Solidi B 174, 295 (1992).

${ }^{27}$ K. Sokolowski-Tinten, J. Bialkowski, A. Cavalleri, D. von der Linde, A. Oparin, J. Meyer-ter-Vehn, and S. I. Anisimov, Phys. Rev. Lett. 81, 224 (1998).

${ }^{28}$ S. I. Anisimov, N. A. Inogamov, A. M. Oparin, B. Rethfeld, T. Yabe, M. Ogawa, and V. E. Fortov, Appl. Phys. A: Mater. Sci. Process. 69, 617 (1999).

${ }^{29}$ V. V. Zhakhovskii, K. Nishihara, S. I. Anisimov, and N. A. Inogamov, JETP Lett. 71, 167 (2000).

${ }^{30}$ D. Bäuerle, Laser Processing and Chemistry, 3rd ed. (SpringerVerlag, Berlin, 2000).

${ }^{31}$ J. König, S. Nolte, and A. Tünnermann, Opt. Express 13, 10597 (2005).

${ }^{32}$ D. E. Aspnes and A. A. Studna, Rev. Sci. Instrum. 49, 291 (1978).

${ }^{33}$ F. Marinozzi, Rev. Sci. Instrum. 71, 4231 (2000).

${ }^{34}$ S. Nakamura and T. Hibiya, Int. J. Thermophys. 13, 1061 (1992).

${ }^{35}$ J. Bonse, K.-W. Brzezinka, and A. Meixner, Appl. Surf. Sci. 221, 215 (2004)

${ }^{36}$ J. P. McDonald, V. R. Mistry, K. E. Ray, S. M. Yalisove, J. A. Nees, and N. R. Moody, Appl. Phys. Lett. 88, 153121 (2006).

${ }^{37}$ P. H. Bucksbaum and J. Bokor, Phys. Rev. Lett. 53, 182 (1984).

${ }^{38}$ A. G. Cullis, Rep. Prog. Phys. 48, 1155 (1985).

${ }^{39}$ R. Cerny, V. Chab, G. Ivlev, E. Gatskevich, and P. Prikryl, Phys. Rev. B 59, 10685 (1999).

${ }^{40}$ J. Bonse, S. M. Wiggins, and J. Solis, J. Appl. Phys. 96, 2352 (2004).

${ }^{41}$ F. J. Pedrotti and L. S. Pedrotti, Introduction to Optics, 2nd ed. (Prentice Hall, Englewood Cliffs, New Jersey, 1993).

${ }^{42}$ J. C. G. de Sande, C. N. Afonso, J. L. Escudero, R. Serna, F. Catalina, and E. Bernabeu, Appl. Opt. 31, 6133 (1992).

${ }^{43}$ Y. Z. Hu, J. T. Zettler, S. Chongsavangvirod, Y. Q. Wang, and E. A. Irene, Appl. Phys. Lett. 61, 1098 (1992).

${ }^{44}$ F. Spaepen and D. Turnbull, Symposium on Laser-Solid Interactions and Laser Processing, AIP Conf. Proc. No. 50 (Materials Research Society, Boston, 1978), pp. 73-83.

${ }^{45}$ P. L. Liu, R. Yen, N. Bloembergen, and R. T. Hodgson, Appl. Phys. Lett. 34, 864 (1979).

${ }^{46}$ J. Bonse, M. Munz, and H. Sturm, IEEE Trans. Nanotechnol. 3, 358 (2004).

${ }^{47}$ M. von Allmen and A. Blatter, Laser-Beam Interactions with Materials, 2nd ed. (Springer-Verlag, Berlin, 1995).

${ }^{48}$ A. G. Cullis, H. C. Webber, and N. G. Chew, Appl. Phys. Lett. 42, 875 (1983).

${ }^{49}$ It should be noted that this flow velocity $u$ at the vacuum boundary can be derived also in an alternative way. According to Sokolowski-Tinten et al. (Ref. 27) it is approximately given by $u \approx c_{0} \ln \left(r_{1} / r_{0}\right)$, where $c_{0}$ is the sound velocity and $r_{0}$ and $r_{1}$ are the solid density and the liquid density near the thermodynamical binodal boundary. Due to the lack of suitable data for $\mathrm{Ge}$, we 
will estimate $u$ directly from our experimental data.

${ }^{50}$ This value of $h=350 \mathrm{~nm}$ has been estimated from an energy balance consideration at fluence close to the melting threshold. It represents an upper limit for the real melt layer thickness; for details of the model see J. Bonse, S. M. Wiggins, and J. Solis, Appl. Phys. A: Mater. Sci. Process. 80, 243 (2005).
${ }^{51}$ Note that both velocities are rather insensitive on the melt layer thickness $h$ : a value of only $50 \mathrm{~nm}$ leads to even smaller velocities of $v_{p}=6 \mathrm{~m} / \mathrm{s}$ and $v_{g}=12 \mathrm{~m} / \mathrm{s}$.

${ }^{52}$ This speed is estimated from the radial distance of $25 \mu \mathrm{m}$ traveled by the fringe pattern from the center in horizontal direction within 1 ns, e.g., in Fig. 8(f). 